\title{
Two approaches to the modelling of chip formation: rheological models and finite element analysis
}

Jüri OLT, DSc. He is a Professor at the Institute of Technology of the Estonian University of Life Sciences. His main researching area are fundamentals of production enginering, dynamics of machining technological systems, materials cutting and design of technological machinery.

JÜRI OLT - Institute of Technology, Estonian University of Life Sciences, Estonia - jyri.olt@emu.ee

OLGA LIIVAPUU - Institute of Technology, Estonian University of Life Sciences, Estonia

- olga.liivapuu@emu.ee

Érkezett: 2019. 09. 24. - Received: 24. 09. 2019. - https://doi.org/10.14382/epitoanyag-jsbcm.2019.30

\begin{abstract}
Metal cutting is a complex process in which several mechanisms are at work simultaneously. The mathematical modelling allows carrying out research into the optimization of machining conditions. Usually, the study of cutting process is very complicated, because the material removal process is conducted in an extremely adverse environment with high temperature and pressure in the zone of cutting. Therefore, the main goal of these studies is to establish a theory that will predict chip formation dynamics, cutting forces, temperature, tool wear, which allows to solve practical problems. This work examines the simulation of chip formation during the process of cutting. The aim of the present research is to compare models based on rheological properties of metals with 2D Finite Element Models of chip formation process.

Keywords: chip formation, elastic and plastic deformation, rheological models, finite element method

Kulcsszavak: forgácsképzôdés, rugalmas és képlékeny alakváltozás, reológiai modellek, véges elemek módszere
\end{abstract}

\section{Introduction}

The chip formation process proceeds with a very significant relative deformation of material, reflecting the high deformation rate in a comparatively small zone. The mathematical modelling will play very important role in increasing the awareness of the cutting process and reducing the number of experiments which are frequently used for a selection of different cutting parameters, tool design, machinability evaluation, etc. The chip formation mechanism in the orthogonal metal cutting process, is the focus in the present research. To simulate this process, we compare two approaches: description of the physical properties of the deformed metal using discrete complex mechanical (rheological) models and finite element models.

The study of the rheological properties of materials is widely used by engineers, physicists, chemists and mathematicians. The physical properties of deformed metal can be described in the form of an adequate mechanical model, constructed as a composition of mechanical elements such as elasticity, viscosity and plasticity [1]. Considering combinations of linear elastic springs, linear viscous dash-pots and elements of plasticity one can build a model which gives an observable perspective reflecting the nature of material removal process, changes in deformation and stresses of material and their dependence on the structure [2], [3], [4]. Many real metals combine behaviors of all these elements and it is possible to consider the stressstrain state of metal under the action of external loads. The rheological models in the form of non-periodic diagrams allow to describe new properties that reflect the process of deformation and destruction of a workable material in the process of cutting. Increasing number of elements will allow to construct more accurate model describing the response of real materials. But in this case there are more material parameters need to be evaluated by experiments, usually, it might be very difficult, if not an impossible, task.

Finite element method (FEM) is most frequently used in metal cutting analysis. In recent years, FEM has become the main tool for simulating metal cutting processes. It provides appropriate approach to visualize cutting process, because it allows to predict various characteristics of the metal cutting such as cutting forces, temperatures, stresses, chip types and shapes, etc. [5-8].

\section{Application of rheological models to the chip formation process}

Metal cutting is a complicated mechanical process, which includes plastic deformation and destruction of metal in the local zone, friction in interaction of the cutting tool with workable material. In accordance with the results of previous studies [8], this interaction accompanied with flat chip formation was examined in two stages up to the moment of the formation of shaving, i.e. the deformation to the shear plane and the shift of the element of small thickness on the plane of shift.

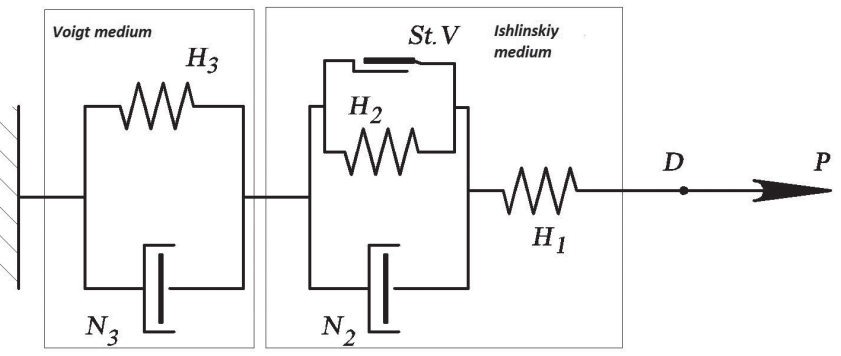

Fig. 1 The model for a mechanical system in the chip formation process 1. ábra A forgácsképzödési folyamat mechanikai rendszerének modellje 
We describe the process of chip formation with the help of the rheological model proposed by Veitz and Maksarov [9]. This model is constructed as combination of two rheological bodies connected in series: the elastic-ductile-plastic medium of Ishlinskiy type [2], [3], [4] and viscoelastic Voigt medium with the delay of deformation (see Fig. 1). The four-element rheological model of Ishlinskiy most fully reflects the dynamics of plastic deformation and destruction of a solid body in the cut off layer of metal. The force $\boldsymbol{P}$ applied to the point $D$ imitates by the linear elastic spring element $H_{1}$ the elastic instantaneous deformation, formed in front of the area of plastic deformation in the zone of chip formation. The next part of this model imitates the prolonged viscoelastic deformation with the help of the spring $\mathrm{H}_{2}$ and the dash-pot $\mathrm{N}_{2}$ connected in parallel. After reaching the yield stress $\sigma_{y}$ which imitates St. Venant element St. $V$. the instantaneous residual deformation appears. This leads to the shaping of local segments of shift in the primary deformation zone of the cut off layer. At this stage, permanent, irreversible deformation begins [10], [11]. The Voigt model, which consists of the spring $\mathrm{H}_{3}$ and the dashpot $N_{3}$ in parallel, describes movement of the shift of material foreword in the direction of tool movement and prolonged viscoelastic deformation.

The process describing relation of brittle and ductile fracture of material is presented in (Fig. 1). Indeed, we can assume that the brittle fracture corresponds to break of elastic external element $H_{1}$ and viscous fracture corresponds to the break of elastic internal element $\mathrm{H}_{2}$ which is preceded by the relative shift of viscous element $N_{2}$ [3]. Elements of mechanical system can be considered in relation to the direction of deformation with the input and output, the direction of the pointer in the (Fig. 1) indicates the direction of deformation of the system.

The differential constitutive relations between stress and strain for the model describing the process of chip formation can be presented in the form of the following rheological equation [12]:

$a_{1} \ddot{\sigma}+a_{2} \dot{\sigma}+a_{3}\left(\sigma \pm \sigma_{y}\right)=a_{4} \ddot{\varepsilon}+a_{5} \dot{\varepsilon}+a_{6} \varepsilon$

where coefficients $a_{i}(i=1, \cdots, 6)$ depend on stiffness coefficients and coefficients of linear resistance, $\sigma_{y}$ is a yield stress.

The solutions and analysis of this rheological equation were studied in [10]. We derive the relations between coefficients of the rheological equation (1) and the stiffness and viscosity of material of the mechanical system. We write the equation (1) in the short-hand notation using integral formulation and the Laplace transform. For the Ishlinskiy model we have

$$
Z_{I}(p)=\frac{p}{c_{1}}+\frac{p}{\beta_{2} \cdot p+c_{2}}=\frac{\beta_{2} p^{2}+\left(c_{1}+c_{2}\right) \cdot p}{\beta_{2} c_{1} \cdot p+c_{1} c_{2}}
$$

where the properties of elastic elements $H_{1}$ and $H_{2}$ of the Ishlinskiy model are described by stiffness coefficients $c_{1}$ and $c_{2}$ the dash-pot element $N_{1}$ is characterized by the coefficient of linear resistance $\beta_{2}$ and $p^{i}=\frac{\partial^{i}}{\partial t^{i}}$ are the linear differential operators.
For the model of Voigt, the operator resistance takes the form

$$
z_{V}(p)=\frac{p}{\beta_{3} \cdot p+c_{3}}
$$

where $\mathrm{H}_{3}$ and $\mathrm{N}_{3}$ are described by the stiffness coefficient $c_{3}$ and $\beta_{3}$ the coefficient of linear resistance respectively.

The entire model describing the process of chip formation can be presented in the following form

$z(p)=p \cdot \frac{\beta_{2} \beta_{3} \cdot p^{2}+\left(\beta_{2} c_{1}+\beta_{2} c_{3}+\beta_{3} c_{1}+\beta_{3} c_{2}\right) \cdot p+\left(c_{1} c_{2}+c_{2} c_{3}+c_{1} c_{3}\right)}{\beta_{2} \beta_{3} c_{1} \cdot p^{2}+\left(\beta_{2} c_{1} c_{3}+\beta_{3} c_{1} c_{2}\right) \cdot p+c_{1} c_{2} c_{3}}$

In order to simplify the calculations, we introduce the following notations for the coefficients of differential operator $p$ and free terms in the numerator and denominator of equation (2):

$$
\left\{\begin{array}{c}
a_{1}=\beta_{2} \beta_{3} \\
a_{2}=\beta_{2} c_{1}+\beta_{2} c_{3}+\beta_{3} c_{1}+\beta_{3} c_{2} \\
a_{3}=c_{1} c_{2}+c_{2} c_{3}+c_{1} c_{3} \\
a_{4}=\beta_{2} \beta_{3} c_{1} \\
a_{5}=\beta_{2} c_{1} c_{3}+\beta_{3} c_{1} c_{2} \\
a_{6}=c_{1} c_{2} c_{3}
\end{array}\right.
$$

Then the operator resistance can be expressed in the form [3]

$$
z(p)=p \cdot \frac{a_{1} \cdot p^{2}+a_{2} \cdot p+a_{3}}{a_{4} \cdot p^{2}+a_{5} \cdot p+a_{6}}
$$

it means that returning to the space of originals we obtain the rheological equation (1), which define the relation between stress and deformation of the mechanical system.

To motivate the adequacy of the rheological model (1) we assume that the deformation in time is supposed to be known and solve this equation for the stress. We substitute $\sigma$ in the equation (1) by a new variable $x=a_{3}\left(\sigma \pm \sigma_{y}\right)$ and get

$\ddot{x}+2 \gamma \dot{x}+\omega_{0}^{2} x=f(t)$,

where

$\omega_{0}=\sqrt{\frac{a_{3}}{a_{1}}}=\sqrt{\frac{c_{1} c_{2}+c_{2} c_{3}+c_{1} c_{3}}{\beta_{2} \beta_{3}}}$ is a frequency,

$\gamma=\frac{a_{2}}{2 a_{1}}=\frac{\beta_{2} c_{1}+\beta_{2} c_{3}+\beta_{3} c_{1}+\beta_{3} c_{2}}{2 \beta_{2} \beta_{3}}$ is a dumping constant,

$f(t)=f(\varepsilon, \dot{\varepsilon}, \ddot{\varepsilon})=\frac{a_{3} a_{4} \cdot \ddot{\varepsilon}+a_{3} a_{5} \cdot \dot{\varepsilon}+a_{3} a_{6} \varepsilon}{a_{1}}$.

Let us denote the vibration frequency by $\omega=\sqrt{ } \gamma^{2}-\omega_{0}^{2}$ The solution of homogeneous part of equation (3) for oscillation mode is

$$
x(t)=e^{-\gamma t}(A \cos \omega t+B \sin \omega t), \quad \text { for } \gamma^{2}-\omega_{0}^{2}<0,
$$

and for relaxation mode

$$
x(t)=A e^{(\omega-\gamma) t}+B e^{(-\omega-\gamma) t}, \quad \text { for } \gamma^{2}-\omega_{0}^{2}>0 .
$$

The general solution of equation (3) for relaxation mode is

$x(t)=e^{-\gamma t}\left[\frac{1}{2} e^{\omega t} \cdot \int \frac{f(t) e^{(\gamma-\omega) t}}{\omega} d t-\frac{1}{2} e^{-\omega t} \cdot \int \frac{f(t) e^{(\gamma+\omega) t}}{\omega} d t+A e^{\omega t}+B e^{-\omega t}\right]$

With a fixed value of deformation $\varepsilon=\varepsilon_{0}=$ const function $f(t)$ can be expressed as follows

$$
f(t)=\frac{a_{3} a_{6} \varepsilon_{0}}{a_{1}}
$$

the phenomenon of stress relaxation is characteristic for a majority of real materials, and is consisting in a gradual decrease of stress in the medium, if the deformation is invariable. 
After transition from variable $x$ to variable $\sigma$ and taking into account initial conditions $\sigma(0)=\sigma_{0}$ and $\dot{\sigma}(0)=$ const an equation describing stress relaxation takes the following form

$$
\begin{aligned}
& \sigma(t)=\sigma_{y}+\frac{a_{6} \varepsilon_{0}}{a_{1}\left(\gamma^{2}-\omega^{2}\right)}+\frac{1}{2 \omega}\left[\left(\sigma_{0}-\sigma_{y}\right)\left((\gamma+\omega) e^{\omega t}+(\omega-\gamma) e^{-\omega t}\right)-\right. \\
& \left.-\frac{a_{6} \varepsilon_{0}}{a_{1}}\left(\frac{1}{\gamma-\omega} e^{\omega t}-\frac{1}{\gamma+\omega} e^{-\omega t}\right)+\dot{\sigma}(0)\left(e^{\omega t}-e^{-\omega t}\right)\right] \cdot e^{-\gamma t}
\end{aligned}
$$

From equation (5) we can conclude that for our rheological model stress relaxes to its equilibrium with two relaxation times $\tau_{1}=\frac{1}{\gamma-\omega}$ and $\tau_{2}=\frac{1}{\gamma+\omega}$. We get the following expression for the for the rates of relaxation $\gamma-\omega$ and $\gamma+\omega$ in the terms of stiffness coefficients $c_{i}$ and coefficients of linear resistance $\beta_{i}$ :

$\gamma \pm \omega=\frac{a_{2}}{2 a_{1}} \pm \sqrt{\frac{a_{2}^{2}-4 a_{1} a_{3}}{4 a_{1}^{2}}}=\frac{1}{2 a_{1}}\left(a_{2} \pm \sqrt{a_{2}^{2}-4 a_{1} a_{3}}\right)=$ $=\frac{1}{2 \beta_{2} \beta_{3}}\left[\left(\beta_{2} c_{1}+\beta_{2} c_{3}+\beta_{3} c_{1}+\beta_{3} c_{2}\right) \pm\right.$

$\left.\pm \sqrt{\left(\beta_{2} c_{1}+\beta_{2} c_{3}+\beta_{3} c_{1}+\beta_{3} c_{2}\right)^{2}-4 \beta_{2} \beta_{3}\left(c_{1} c_{2}+c_{2} c_{3}+c_{1} c_{3}\right)}\right]$.

It follows from proposed rheological model in the zone of primary plastic deformation occur processes which generate instability of chip formation. The analysis of the developed plastic deformation of metal shows that this process has wavelike nature. At the moment which corresponds to the implementation of plastic deformation in the zone of plastic contact of chip with the tool, which reflects the elastic reaction of the workable material, the cut off layer undergoes the secondary deformation. Our theoretical studies were confirmed by experimental data which were conducted in the broader band for back rake angle $\alpha$ from $-5^{\circ}$ to $25^{\circ}$ and for different values of cutting speed. It was determined in [2] that the form of chip by processing steel AISI 45 is following:

- discontinuous chip for $\alpha=-5^{\circ}$ and cutting speed $10 \mathrm{~m} / \mathrm{min}$;

- built-up edge chip for $\alpha=25^{\circ}$ and cutting speed $25 \mathrm{~m} / \mathrm{min}$;

- continuous chip for $\alpha=10^{\circ}$ and cutting speed $75 \mathrm{~m} / \mathrm{min}$.

It confirms our studies on the origin of the primary relaxation phenomena, which induce the origination of the self-oscillating process by turning materials.

\section{Finite element analysis of chip formation process and description of simulation model}

The basis of all finite elements methods involves dividing the body into an equivalent system of small finite elements for which the relevant variables and quantities are determined only at the nodes of the elements. This procedure is called discretization or meshing. The accuracy of the solution depends on the total number of elements used and their variation in size and type. The elements must be made small enough to give relevant results and large enough to reduce time required for the simulation. In recent years, numerous special-purpose and general-purpose finite element packages have been used for metal cutting analysis: Deform 2D/3D, MSC.Marc., Thirdwave
AdvantEdge, Abaqus, Ls-Dyna, etc. In modeling the plastic material flow there are three main formulations for simulation of metal cutting Eulerian, Lagrangian and Arbitrary LagrangianEulerian (ALE). The last one combines the best features of Lagrangian and Eulerian formulations [13]. In this case, the mesh is not attached to the material and it can move to avoid distortion and update the free chip geometry. The mesh follows the material flow and problem is solved for displacements in Lagrangian step, while the mesh is regenerated and problem for velocities is solved in Eulerian step.

The thermo-mechanical modeling of flow stress of workpiece material plays very important role in metal cutting simulation. In finite element models flow stress is an instantaneous yield stress which depends on strain, strain rate and temperature. To describe the material flow during the cutting process an elastoviscoplastic description of the material behavior can be written in the form of constitutive models:

$\sigma=f(\varepsilon, \dot{\varepsilon}, T)$,

where $\sigma$ is the Von-Mises stress, $\varepsilon$ is the plastic strain, $\dot{\varepsilon}$ is the generalized strain rate and $T$ is the temperature. The most widely used is Johnson-Cook material constitutive model. This model is based on torsion and dynamic Hopkinson bar test over a wide range of strain rates and temperatures [14]. This constitutive equation has the following form:

$\sigma=\left(A+B \varepsilon^{n}\right)\left(1+C \ln \frac{\dot{\varepsilon}}{\dot{\varepsilon}_{0}}\right)\left(1-\left(\frac{T-T_{r}}{T_{m}-T_{r}}\right)^{m}\right)$

The elastic-plastic term representing strain hardening is in the first parenthesis. The coefficient $A$ is the yield strength value when the stressing rate is low, $B$ is the hardening modulus and $n$ is the hardening coefficient. The viscosity term, where $C$ is the strain rate sensitivity coefficient, is in the parenthesis, it shows that flow stress of material increases when material is exposed to high strain rates. The last one is temperature softening term. $T$ is the instantaneous temperature, $T_{r}$ is the room temperature, $T_{m}$ is the melting temperature of a given material and $m$ is the thermal softening coefficient. JohnsonCook constitutive model assumes that flow stress is affected by strain, strain rate and temperature independently.

In the present research we consider the finite element model used for the plane-strain orthogonal metal cutting simulation, which is based on the Lagrangian formulation in Third Wave Systems AdvantEdge simulation software. AdvantEdge integrates finite element numerics and material modeling appropriate for metal cutting. The software has comprehensive library of materials, input interface allows to set cutting parameters (feed rate, depth of cut, length of cut, cutting speed and initial temperature of the workpiece) as well as tool and workpiece geometries. Other parameters of cutting are fixed automatically, the user control on material parameters and solver are not allowed. The material model considers elasticplastic strains, strain rate and has an isotropic power law for strain hardening. In AdvantEdge a staggered method is utilized for the purpose of coupling the mechanical and heat transfer equations. Geometrically identical meshes for the mechanical and thermal models are used. An isothermal mechanical step is taken first, it is followed by a rigid transient thermal step 
with constant heating from plastic work and friction. The finite deformation formulation uses a six-noded quadratic triangle elements. As the cutting layer transforms into chip, it separates from the workpiece splitting the nodes. The separation of nodes is achieved by continuous remeshing, the software enables element distortion around the cutting edge by updating the mesh periodically both refining large elements and coarsening small elements.

\section{Conclusions}

The rheological model in the form of a series connection of elastic-ductile-plastic relaxing medium of Ishlinskiy and the medium of Voigt describes the mechanical properties of chip formation process, but does not contain the evaluation of cutting temperature. The analysis of rheological model shows that instability of the process of cutting is caused by the wavelike nature of the plastic deformation and destruction of metal. Within the generalized rheological model of the chip formation process, material deformed in the cut off layer reflects the relaxation effect inherent in stress. Stress relaxes to its equilibrium value not according to the simple exponential law, but with a significantly more complicated law, with two relaxation times $\tau_{1}=\frac{1}{\gamma-\omega}$ and $\tau_{2}=\frac{1}{\gamma+\omega}$ At the moment which corresponds to the completion of plastic deformation in the zone of plastic contact of chip with the tool and which also reflects the elastic reaction of the workable material in the process of cutting, the cut off layer undergoes a second deformation. The instability of chip formation in the primary zone of the plastic deformation process is the basic reason for the appearance of instability within the process of cutting in the closed dynamic system of a machine tool. The analysis of the rheological equation (1) proves that the model enables an adequate description of the deformation process of chip formation and determination of the criteria to its transition to a non-stable mode, provoking self-excited oscillation.

High temperature in the cutting zone hostilely affects the strength, hardness and wear resistance of the cutting tool. Therefore, estimation of cutting temperature is a decisive aspect in the study of metal cutting. The future work on the present rheological model will focus on adding the temperature parameters into the rheological equation (1) describing the process of chip formation. To this end, will use the rheological model proposed by Malyshev [15]. For this model it is assumed that the plastic deformation of the metal in the shear zone can be considered as the result of compression of the metal layer, taking into account the friction of the chips on the front surface of the tool and the cutting temperature.

With the advent of efficient software packages, FEM has become one of the most powerful tools for the simulation and analysis of cutting process. This allows studying the cutting process in greater detail than possible in experiments. FEM simulation takes into account the material properties better than analytical models. FEM models allow to simulate the interaction of chip and tool in different forms. In spite of many advantages of FEM models, it is difficult to simulate the morphology of cutting chips. The continuous chip is often considered as more preferable, because it generates stable cutting forces. Moreover, it is easiest for simulation codes, but during experiments the sharp edged continuous chip that comes out at a high speed may be dangerous to the operator and may lead to unpredictable damage of the cutting tool. The majority of finite element codes simulates only continuous chips.

\section{References}

[1] Rybin, V.V. (1986) Large plastic deformation and fracture of metals. Metallurgiya, Moscow, $318 \mathrm{p}$.

[2] Maksarov, V., Olt, J., Laatsit, T, Leemet, T. (2008) Physical argumentation of deformation process potential control while cutting heavily processed material. Proceedings of 6th International DAAAM Baltic Conference INDUSTRIAL ENGINEERING, Tallinn, pp. 263-268.

[3] Maksarov, V.; Khalimonenko, A.; Olt, J. (2017). Managing the process of machining on machines on the basis of dynamic modelling for a technological system. Építőanyag - Journal of Silicate Based and Composite Materials, 69 (2), pp. 66-71, https://doi.org/10.14382/epitoanyag-jsbcm.2017.12.

[4] Olt, J., Liyvapuu, A., Madissoo, M., Maksarov, V. (2016) Dynamic simulation of chip formation in the process of cutting. Int. J. Mater. Prod. Tec. Vol. 53, No 14, pp. 1-14, https://doi.org/10.1504/IJMPT.2016.076363

[5] Arrazola, P. J., Ugarte, D., Montoya, J., Villar, A., Marya, S. (2005) Finite element modeling of chip formation process with Abaqus/Explicit 6.3. [Performance].VIII International Conference on Computational Plasticity, Barcelona, URL:

http://congress.cimne.upc.es/complas05/admin/Files/FilePaper/p173.pdf

[6] Boros, B., Csörgö, A., Hidas, A., Kotnyek, B., Szabó, A., Kossa, A., Stépán, G. (2017) Two-Dimensional Finite Element Analysis of Turning Processes. Periodica Polytechnica Mechanical Engineering. Vol. 61, No 1, pp. 44-54, https://doi.org/10.3311/PPme.9283

[7] Öpöz, T.T., Chen, X. (2016) Chip formation mechanism using finite element simulation. J.Mech.Eng. Vol. 62, pp. 312-325, https://doi.org/10.5545/sv-jme.2016.3523

[8] Watmon, T. B., Xiao, D., Peter, O.-O. (2016) Finite element analysis of orthogonal metal machining. Int. J. Sci. Res. Inn. Tech. Vol. 3, No 2, pp. 46-55.

[9] Veitz, V., Maksarov, V. (2000) Dynamics and control of the process of chip formation with cutting-edge mechanical processing. NWPI, SantPetersburg, $225 \mathrm{p}$.

[10] Tärgla, T., Olt, J., Liivapuu, O. (2018) Simulation of plastic deformation and destruction in the process of chip formation. Matec Web of Conferences. Vol. 211, pp. 1-5, https://doi.org/10.1051/matecconf/201821117007

[11] Liivapuu, O., Olt, J., Tärgla, T. (to appear) Comparison between finite element analysis and rheological models for chip formation. Acta et Commentationes Universitatis Tartuensis de Mathematica.

[12] Olt, J., Maksarov, V. (2015) Development of chatter-resistant system of cutting tool. Proceedings of the DAAAM symposium. pp. 0223-0228, https://doi.org/10.2507/26th.daaam.proceedings.03

[13] Hughes, T. J. R. (2000) The finite element method. Linear static and dynamic finite element analysis. Prentice-hall, New Jersey, 704. p.

[14] Johnson, G.R., Cook, W. H. (1983) A constitutive model and data for metals subjected to large strains, high strain-rates and high temperatures. Proceedings of the 7th International Symposium of Ballistics. pp. 541-547.

[15] Malyshev, V.I. (2011) Essays on the history of science on cutting materials. TGU, Tolyatti, $314 \mathrm{p}$.

Ref.:

Olt, Jüri - Liivapuu, Olga: Two approaches to the modelling of chip formation: rheological models and finite element analysis Építőanyag - Journal of Silicate Based and Composite Materials, Vol. 71, No. 5 (2019), 173-176. p. https://doi.org/10.14382/epitoanyag-jsbcm.2019.30 